\title{
Interactions between Medial Temporal Lobe, Prefrontal Cortex, and Inferior Temporal Regions during Visual Working Memory: A Combined Intracranial EEG and Functional Magnetic Resonance Imaging Study
}

\author{
Nikolai Axmacher, ${ }^{1,2}$ Daniel P. Schmitz, ${ }^{1,3}$ Tobias Wagner, ${ }^{1}$ Christian E. Elger, ${ }^{1,2}$ and Juergen Fell ${ }^{1}$ \\ ${ }^{1}$ Department of Epileptology, University of Bonn, and ${ }^{2}$ Life and Brain Center of Academic Research, D-53105 Bonn, Germany, and ${ }^{3}$ Department of Applied \\ Mathematics, University of Applied Sciences Remagen, 53424 Remagen, Germany
}

It is a fundamental question whether the medial temporal lobe (MTL) supports only long-term memory encoding, or contributes to working memory (WM) processes as well. Recent data suggest that the MTL is activated whenever multiple items or item features are being maintained in WM. This may rely on interactions between the MTL or the prefrontal cortex (PFC) and content-specific areas in the inferior temporal (IT) cortex. Here, we investigated the neural mechanism through which the MTL, PFC, and IT cortex interact during WM maintenance. First, we quantified phase synchronization of intracranial EEG data in epilepsy patients with electrodes in both regions. Second, we used directional coupling analysis to study whether oscillatory activity in the IT cortex drives the MTL or vice versa. Finally, we investigated functional connectivity in functional magnetic resonance imaging data of healthy subjects with seeds in the MTL and PFC. With increasing load, EEG phase synchronization between the IT cortex and anterior parahippocampal gyrus and within the MTL increased. Coupling was bidirectional in all load conditions, but changed toward an increased top-down (anterior parahippocampal gyrus $\rightarrow$ IT) coupling in the high gamma range $(51-75 \mathrm{~Hz})$ with increasing load. Functional connectivity between the MTL seed and the visual association cortex increased with load, but activity within the MTL and the PFC correlated with fewer voxels, suggesting that more specific neural networks were engaged. These data indicate that WM for multiple items depends on an increased strength of top-down control of activity within the IT cortex by the MTL.

Key words: working memory; hippocampus; synchronization; directional coupling; functional connectivity; intracranial EEG

\section{Introduction}

Working memory (WM) refers to the short-term maintenance and manipulation of items (Baddeley and Hitch, 1974). Recently, several studies showed that the medial temporal lobe (MTL) is required in WM tasks involving multiple items or conjunctions of item features (Aggleton et al., 1992; Hannula et al., 2006; Olson et al., 2006; Piekema et al., 2006; Axmacher et al., 2007), in line with the relational memory theory of the hippocampus (Cohen and Eichenbaum, 1993). The hippocampus communicates with the neocortex mainly via polymodal archicortical regions within the anterior parahippocampal gyrus (aPHG) (Witter and Amaral, 1991). These structures, in turn, receive modality-specific information from higher-order sensory regions such as the inferior temporal (IT) neocortex. Interactions between these IT regions, MTL, and prefrontal cortex (PFC) are considered crucial

Received Dec. 6, 2007; revised May 29, 2008; accepted June 2, 2008.

This work was supported by Volkswagen Foundation Grant I-79878. We thank Michael Cohen for critically reading an earlier version of this manuscript, Pascal Fries for helpful suggestions, and Hannes Osterhage and Klaus Lehnertz for help with the directional coupling analysis.

Correspondence should be addressed to Dr. Nikolai Axmacher, Department of Epileptology, University of Bonn, Sigmund Freud Strasse 25, D-53105 Bonn, Germany. E-mail: nikolai.axmacher@ukb.uni-bonn.de.

DOI:10.1523/JNEUROSCI.1778-08.2008

Copyright $\odot 2008$ Society for Neuroscience $\quad$ 0270-6474/08/287304-09\$15.00/0 for various memory tasks: IT-MTL interactions may support the temporal organization of multiple assemblies, each representing individual items (Jensen and Lisman, 2005). As a result, these representations may become sparser and more selective, similar to effects during long-term memory (LTM) encoding (Reddy and Kanwisher, 2006; Axmacher et al., 2008). The experimental evidence for the relationship between memory processes and the sparseness of representations is described in detail in the supplemental material (available at www.jneurosci.org).

Interactions between the IT cortex, PFC, and parietal cortex likely support WM maintenance via an attention-based mechanism (Fuster, 1995), which involves the selective enhancement of relevant and suppression of irrelevant activity (Hopf et al., 2006) and may thus also contribute to sparser representations. Previous functional connectivity studies of functional magnetic resonance imaging (fMRI) data revealed inconsistent results concerning load-dependent interactions between PFC and IT cortex during multi-item WM (Fiebach et al., 2006; Rissman et al., 2008). Although these studies demonstrate PFC-IT and MTL-IT interactions, direct PFC-MTL connectivity has also been observed (Petersson et al., 2006; Rissman et al., 2008).

To test the hypothesis that interactions between the IT cortex, MTL, and PFC support multi-item WM, we reanalyzed data 
mostly based on a previously published study (Axmacher et al., 2007) using a modified Sternberg paradigm with a variable number of consecutively presented faces. First, we calculated phase synchronization (Lachaux et al., 1999; Fell et al., 2001; TallonBaudry et al., 2001) between the hippocampus, aPHG, and IT cortex in intracranial EEG data from epilepsy patients. We hypothesized that synchronization would increase linearly with the number of items to be maintained, indicating enhanced communication between aPHG and IT cortex (Rissman et al., 2008). Furthermore, the direction of coupling was analyzed using a phase-modeling approach (Rosenblum and Pikovsky, 2001). The sign of the directionality index indicates whether oscillatory activity in the IT cortex drives the aPHG, suggestive of bottom-up information flow, or whether the aPHG controls the IT cortex by top-down modulation.

Although the placement of intracranial electrodes follows purely clinical considerations and usually does not cover widespread brain regions, this limitation does not apply to fMRI. Thus, we repeated the same experiment using fMRI in healthy control subjects. Load-dependent functional interactions as well as network extensions were calculated using the beta correlation method for quantifying functional connectivity (Rissman et al., 2004).

\section{Materials and Methods \\ Subjects}

iEEG. Thirteen patients with pharmacoresistant temporal lobe epilepsy (three women; mean age $\pm S D, 38.3 \pm 12.3$ years; handedness, 11 right, 2 left) participated in the study. In nine patients, unilateral hippocampal sclerosis was confirmed histologically. From the others, one had a unilateral isolated amygdala lesion, two others had no apparent MRI lesions and one had a unilaterally accentuated limbic pathology. Recordings were performed from 2004 to 2007 at the Department of Epileptology, University of Bonn, Germany. Twelve patients had bilateral hippocampal depth electrodes, one had a single electrode in the right hippocampus and an extrahippocampal (temporo-occipital) seizure onset zone. Eight of these patients had additional strip electrodes with a total of eight electrode contacts at temporal basal sites. No seizure occurred within $24 \mathrm{~h}$ before the experiment.

$f M R I$. Twenty-three healthy subjects (12 women; mean age $\pm \mathrm{SD}$, $30.0 \pm 10.4$ years; handedness, 22 right, 1 left) participated in the study. They were recruited from the University of Bonn as well as via newspaper.

Both studies were approved by the local medical ethics committee, and all patients and subjects gave written informed consent.

\section{Experimental paradigm}

For a complete description of the paradigm, please refer to Axmacher et al. (2007). In brief, we used a modified Sternberg WM paradigm with serial presentation of items, which allows for parametric modulation of the WM load. Subjects had to memorize one, two, or four black and white photographs of unknown male and female faces (total of 126 male and 126 female faces). Trial timing differed slightly for the intracranial EEG (iEEG) and fMRI experiments to account for different temporal signal characteristics; supplemental Figure 1 (available at www. jneurosci.org as supplemental material) provides an overview of the paradigm in the two conditions. The experiment was run in a single block containing a total of 108 trials, i.e., 36 in each condition (one, two, or four faces). Only trials with a correct response were taken into account.

\section{Recordings}

iEEG. Multicontact depth electrodes and temporal basal strips were inserted for diagnostic purposes using a computed tomography-based stereotactic insertion technique (Van Roost et al., 1998). The location of depth electrode contacts was ascertained by MRI in each patient and was classified as either hippocampal, anterior parahippocampal, or other. Because our methods cannot clearly separate perirhinal and entorhinal generators, we use the term anterior parahippocampal gyrus without indicating an integrated processing stage. Depth EEG was referenced to linked mastoids, recorded at a sampling rate of $1000 \mathrm{~Hz}$, and bandpass filtered [0.01 Hz (6 dB/octave) to $300 \mathrm{~Hz}$ (12 dB/octave)].

fMRI. Sixteen axial slices were collected at 1.5T (Avanto; Siemens). We collected $780 \mathrm{~T} 2{ }^{\star}$-weighted, gradient echo echoplanar imaging scans, including five initial scans that were discarded to achieve steady-state magnetization (slice thickness, $3 \mathrm{~mm}$; interslice gap, $0.3 \mathrm{~mm}$; matrix size, $64 \times 64$; field of view, $192 \mathrm{~mm}$; echo time, $40 \mathrm{~ms}$; repetition time, 3200 $\mathrm{ms})$. Thereafter, we acquired a sagittal T1-weighted magnetizationprepared rapid-acquisition gradient echo sequence for each subject for anatomical localization (number of slices, 160; slice thickness, $1 \mathrm{~mm}$; interslice gap, $0.5 \mathrm{~mm}$; matrix size, $256 \times 256$; field of view, $256 \mathrm{~mm}$; echo time, $3.93 \mathrm{~ms}$; repetition time, $1660 \mathrm{~ms}$ ).

\section{Analyses}

IEEG data. EEG trials were visually inspected for artifacts (e.g., epileptiform spikes), and $12.6 \%$ of all trials were excluded from analysis. From the contralateral (nonfocal) electrode in each patient, we selected the hippocampal and the anterior parahippocampal contact with the maximal slope of the DC potential, because this measure likely corresponds to working memory maintenance (Axmacher et al., 2007). In the IT cortex, we visually selected the temporal basal electrode with the most pronounced N200 component, which is the most prominent event-related potential (ERP) component within the IT cortex corresponding to face processing (Allison et al., 1994).

EEG trials were filtered in the frequency range from 2 to $100 \mathrm{~Hz}(2$ $\mathrm{Hz}$ steps) by continuous wavelet transforms implementing Morlet wavelets of five-cycle length (Daubechies, 1990). The filtered signals result from the time convolution of original signals and the complex wavelet function. To avoid edge effects, the trials entering the wavelet transform were segmented from -4 to $7.5 \mathrm{~s}$ with respect to presentation of the last stimulus in each trial. An interval of $4 \mathrm{~s}$ at the beginning and the end of the trials was afterward discarded. For statistical analyses, phase-synchronization values were averaged for nonoverlapping successive time windows of $500 \mathrm{~ms}$ duration from 0 to $3500 \mathrm{~ms}$ after the onset of the last stimulus in each trial. Afterward, values were normalized with respect to the prestimulus time window from -200 to $0 \mathrm{~ms}$ separately for each subject and each filter frequency. For graphical depiction, power values were transformed into $\mathrm{dB}$ scale (10 $\left.\times \log _{10}\right)$. Because of the intrinsic logarithmic frequency scaling of the wavelet decomposition, frequencies are not statistically independent if sampled too closely on an equidistant frequency scale. Therefore, the EEG was analyzed in the following spectral bands: theta, 3-8 Hz; alpha, 9-12 Hz; beta $1,13-18 \mathrm{~Hz}$; beta $2,19-25 \mathrm{~Hz}$; gamma 1 , $26-50 \mathrm{~Hz}$; gamma $_{2}, 51-75 \mathrm{~Hz}$; gamma $3,76-100 \mathrm{~Hz}$. Statistical tests were calculated using SPSS software (SPSS). $p$ values in the ANOVAs were Huynh-Feldt corrected for inhomogeneities of covariance when necessary (Huynh and Feldt, 1976).

Directional relationships were analyzed using the directionality index suggested by Rosenblum and Pikovsky (2001). This phase-modeling approach is based on the concept of phase synchronization (Pikovsky et al., 2001) between weakly coupled oscillators and was previously applied to intracranial EEG time series by Osterhage et al. (2007). First, we extracted unwrapped phases from the preselected and prefiltered iEEG trials (4096 ms length) using wavelet transforms based on Morlet wavelets. To eliminate edge effects, the initial and final $1024 \mathrm{~ms}$ were afterward discarded. The center frequencies and band widths of the wavelets were chosen in accordance to the bands described above. Only higher-frequency bands were considered because signal approximation becomes inaccurate for small numbers of oscillation cycles (Rosenblum et al., 2001). In our case, at least 40 cycles contributed to the statistics.

Phase dynamics of two coupled systems can be approximated by maps using Fourier series in dependency of the extracted phases $f_{1,2}\left(\theta_{1,2}, \theta_{2,1}\right)$ (Rosenblum and Pikovsky, 2001). The mutual influence of the systems was quantified by the coefficients. 


$$
c_{1,2}^{2}=\int_{0}^{2 \pi} \int_{0}^{2 \pi}\left(\frac{\partial f_{1,2}}{\partial \theta_{2,1}}\right)^{2} d \theta_{1,2} d \theta_{2,1},
$$

and the normalized directionality of coupling was calculated as

$$
d^{(1,2)}=\frac{c_{2}-c_{1}}{c_{1}+c_{2}}
$$

with $d^{(1,2)} \in[-1,1]$. In the case of positive values, the first system predominantly drives the second (and vice versa). For example, for the analysis of directional coupling between the IT cortex and aPHG, the first system was the IT cortex and the second system the aPHG. Thus, positive values represented IT cortex $\rightarrow$ aPHG (bottom up) coupling, and negative values aPHG $\rightarrow$ IT cortex (top down) coupling.

Very recently, this method was further improved by preprocessing the extracted observably dependent nonuniverse phases $\theta_{1,2}$ into invariant phases $\phi_{1,2}$ (Kralemann et al. 2007). The genuine invariant phases are almost independent of the used extraction method (e.g., Hilbert or Poincaré transform) and thereby observably independent. The theoretical idea is to reconstruct the differential equation of the genuine phase dynamics $\dot{\phi}_{1,2}=\omega_{1,2}+q^{(1,2)}\left(\phi_{1,2}, \phi_{2,1}\right)$, with $\omega_{1,2}$ as frequencies and $q^{(1,2)}$ as coupling functions, from the differential equations for the observably dependent phases $\dot{\theta}_{1,2}=f_{1,2}\left(\theta_{1,2}, \theta_{2,1}\right)$. Following Kralemann et al. (2007), we first preprocessed both extracted phases by applying the transformation for uncoupled oscillators (order of the Fourier series, 48). Subsequently, the maps $f_{1,2}$ were approximated with Fourier series (order, 10) to the phase time series. After the transformation of observed to genuine phases, we achieved the invariant phases $\phi_{1,2}$ and their derivatives. From this, we fitted the coupling function of the genuine phases by Fourier series with coefficients $Q_{n, m}^{(1,2)}$ with $|n| \leq 9,|m| \leq 9$, resulting in $\phi_{(1,2)}=q^{(1,2)}\left(\phi_{1,2}, \phi_{2,1}\right)=\sum_{n, m} Q_{n, m}^{(1,2)} \exp \left(i\left[n \phi_{1,2}+m \phi_{2,1}\right]\right)$. With this map, we calculated the directionality index $d^{(1,2)}$ by replacing the former coefficients $c_{1,2}$ with $C_{1,2}$ $=\left(\sum_{n, m}\left|Q_{n, m}^{(1,2)}\right|^{2}\right)^{1 / 2} / \omega_{1,2}$, where the summations exclude the case of $n=$ $m=0$, and $\omega_{1,2}$ is the reconstructed frequency. This advanced approach is free of a bias using the uncorrected phases.

This method works under the assumption of weakly coupled systems. To avoid directional misinterpretation caused by completely phase synchronized or uncoupled systems, values of $d^{(1,2)}$ were discarded if the simultaneous mean phases coherence (Mormann et al., 2000) was greater than an empirical value of 0.95 (Smirnov et al., 2007) or smaller than 0.05. Finally, the directional indices calculated for each ERP segment were averaged for each patient and condition. For statistical analyses, directionality values were Fisher's $z$ transformed.

$f M R I$. MRIs were processed using SPM2 (www.fil.ion.ucl.ac.uk/ $\mathrm{spm} /$ ). Preprocessing included realignment and unwarping, normalization, spatial smoothing with an $8 \mathrm{~mm}$ Gaussian kernel, and high-pass temporal filtering at $128 \mathrm{~Hz}$. In the analyses, we estimated single-trial parameters of the following regressors: combined encoding and maintenance phase in trials with one, two, or four faces (three boxcar regressors of $12 \mathrm{~s}$ length); retrieval (boxcar regressor of $1.5 \mathrm{~s}$ length); and baseline periods (pauses of $30 \mathrm{~s}$ duration, modeled by $30 \mathrm{~s}$ boxcar regressors). Encoding and maintenance periods were combined for estimation of single-trial parameters to avoid collinearity among these regressors and to improve the reliability of parameter estimates. In our previous study (Axmacher et al., 2007), encoding and maintenance phase were modeled separately. However, beta values were not calculated for single trials, but for all trials of one condition (e.g., for all load 1 trials), which results in a substantially higher accuracy of beta values compared with analyses of individual trials. Incorrect trials were modeled with a separate regressor regardless of memory load.

Correlations between single-trial beta parameter estimates were calculated according to the method proposed by Rissman et al. (2004). For statistical comparisons, correlation values were Fisher's $z$ transformed. We investigated networks with significant functional connectivity based on functional regions of interest in the left hippocampus [Montreal Neurological Institute (MNI) coordinates $-24,-21,-15]$, the left PFC (MNI coordinates $-42,9,30$ ), and the left fusiform cortex (MNI coordinates, $-36,-48,-21)$. These seed regions were selected from the most significant voxel (and the neighboring six voxels) in an MTL cluster showing enhanced activity with increasing memory load during the late maintenance phase (which has been described previously to be most accurate for WM maintenance) (Zarahn et al., 1997; Postle et al., 2000), in a prefrontal cluster showing enhanced activation during the encoding phase, and from a local maximum in the fusiform cortex in a larger inferior temporal cluster (Axmacher et al., 2007).

All figures with fMRI results are displayed using neurological convention (left hemisphere on the left side of the figure). To identify significant correlations, we used an uncorrected threshold of $p<0.001$ (two-tailed). A minimum cluster size of five contiguous voxels was used as an additional threshold in all tests. The number of significantly correlated voxels in the different load conditions was normalized in each subject by the total number of correlated voxels in this subject (across all conditions).

\section{Results}

\section{Behavioral data}

The analyses presented here are based on an extension (two additional patients, data from the IT cortex) of a data set that has been published previously (Axmacher et al., 2007). Similar to these previous data, an analysis of accuracy values in a two-way ANOVA with load as the repeated measure and subject group (extended group of epilepsy patients vs fMRI subjects) as the independent variable revealed significant effects of load $\left(F_{(2,68)}=\right.$ 21.627; $\left.p<10^{-6} ; \varepsilon=0.899\right)$ and subject group $\left(F_{(1,34)}=21.209\right.$; $p<0.0001$ ), but no interaction (numerical results in the extended group of epilepsy patients, mean \pm SEM: $79.45 \pm 3.64 \%$; $77.77 \pm 3.20 \% ; 66.63 \pm 4.84 \%)$. Also in accordance with our previous analyses, we did not find a significant effect of load on reaction times (numerical results in the extended group of epilepsy patients, mean \pm SEM: $1656 \pm 239 \mathrm{~ms} ; 1571 \pm 172 \mathrm{~ms}$; $1578 \pm 179 \mathrm{~ms})$.

\section{Phase synchronization in intracranial EEG}

To investigate the communication of the aPHG with regions in the IT cortex and within the MTL during multi-item WM, we calculated phase synchronization between electrode contacts in the following regions: between the IT cortex and the aPHG and between aPHG and hippocampus (because there are no direct connections between IT cortex and hippocampus, synchronization between these structures was not analyzed). We used a modified Sternberg paradigm with serial presentation of one, two, or four photographs of unknown male and female faces (supplemental Fig. 1, available at www.jneurosci.org as supplemental material) which allowed for a parametric modulation of memory load.

The results for the synchronization between the IT cortex and aPHG are shown in Figure 1. A three-way ANOVA with memory load, time, and frequency band as repeated measures revealed a significant load $\times$ band interaction $\left(F_{(12,84)}=2.582 ; p<0.05 ; \varepsilon\right.$ $=0.451)$. Subsequent two-way ANOVAs with memory load and time as repeated measures in the different frequency bands

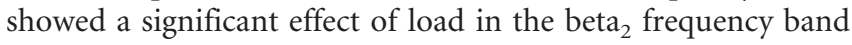
$\left(19-25 \mathrm{~Hz} ; F_{(2,14)}=4.353 ; p<0.05\right)$. This effect was caused by a linear increase in synchronization with load as indicated by a significant linear effect $\left(F_{(1,7)}=6.185 ; p<0.05\right)$. None of the other bands showed an effect of load or a load $\times$ time interaction.

Next, we analyzed synchronization between aPHG and hippocampus (Fig. 2). Although we did not observe a significant load $\times$ band interaction in a three-way $\operatorname{ANOVA}\left(F_{(12,144)}=\right.$ 
$0.619 ; p=0.776 ; \varepsilon=0.738)$, previous findings suggest an important role of aPHG-hippocampal synchronization in the lower gamma frequency range in memory processing (Fell et al., 2001). Indeed, we found a load-dependent increase of $\mathrm{aPH}$ $\mathrm{G}$-hippocampal synchronization in the gamma ${ }_{1}$ frequency band $\left(26-50 \mathrm{~Hz} ; F_{(2,24)}\right.$ $=4.702 ; p<0.05)$ and a linear effect in this range $\left(F_{(1,12)}=7.801 ; p<0.05\right)$. None of the other bands showed an effect of load or a load $\times$ time interaction. Together, these findings indicate that increasing load correlates with enhanced IT-aPHG synchronization in the beta frequency band $_{2}$ and enhanced aPHG-hippocampal synchronization in the gamma ${ }_{1}$ range.

Does the PFC also show loaddependent synchronization with the medial temporal and IT cortices? This question could be investigated in one patient who had 16 electrodes in the PFC, anterior $\mathrm{PHG}$, hippocampus, and inferior temporal cortex of one hemisphere (Fig. $3)$. All 16 electrode contacts showed enhanced average synchronization values in the upper gamma range $(>50 \mathrm{~Hz})$ during processing of multiple items compared with a single item; this effect appeared to be most pronounced for frontal-IT synchronization. In contrast, average synchronization in the lower frequency range decreased for some frontal contacts. To test these effects statistically, we computed three-way ANOVAs with memory load, time, and frequency band as repeated measures across the 16 frontal electrodes (synchronization with the hippocampus, aPHG, and IT cortex). For synchronization with the hippocampus, we observed a significant three-way interaction $\left(F_{(72,1080)}=1.985 ; p<0.0001 ; \varepsilon=\right.$ $0.739)$. Separate two-way ANOVAs in the different frequency bands revealed a significant load $\times$ time interaction effect in the gamma $_{3}$ range $\left(F_{(12,180)}=2.548\right.$; $p=0.010 ; \varepsilon=0.735)$, and a trend for a load effect in this band $\left(F_{(2,30)}=3.200\right.$; $p=0.064 ; \varepsilon=0.866)$. None of the other bands showed a significant load effect or load $\times$ time interaction. For synchronization with the aPHG, we found a significant load $\times$ band interaction $\left(F_{(12,180)}\right.$ $=4.306 ; p<0.0001 ; \varepsilon=0.828$ ). Analyses in the separate bands revealed significant load $x$ time interactions in the $\operatorname{gamma}_{2}\left(F_{(12,180)}=2.265 ; p<0.05 ; \varepsilon=\right.$ $0.827)$ and gamma 3 bands $\left(F_{(12,180)}=\right.$ $1.988 ; p<0.05 ; \varepsilon=0.948)$, but no load effects and no load $\times$ time interaction in any other band. For synchronization with the IT cortex, the three-way ANOVA revealed a significant three-way interaction $\left(F_{(72,1080)}=1.354 ; p<0.05 ; \varepsilon=0.821\right)$.
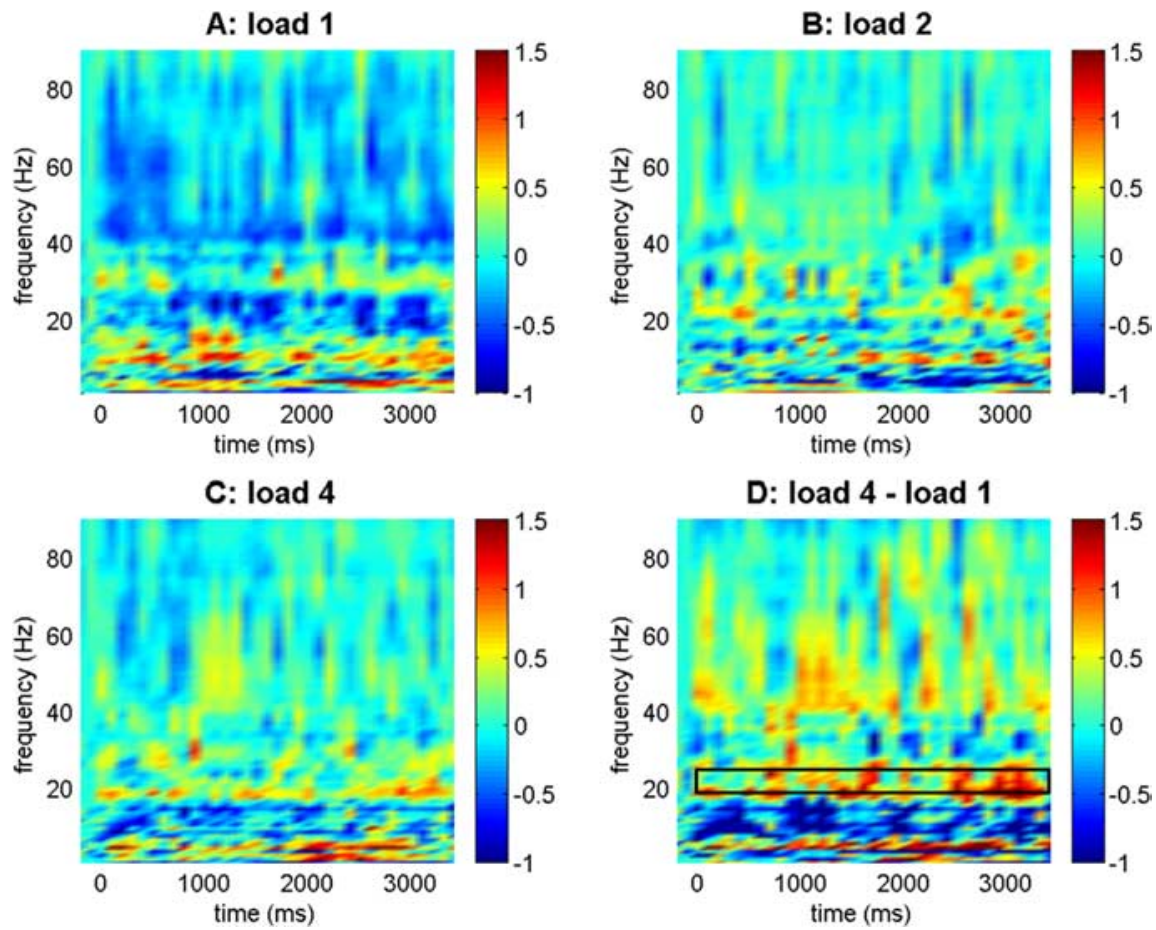

Figure 1. Synchronization between anterior parahippocampal gyrus and inferior temporal cortex. Baseline-normalized synchronization values are indicated for the different load conditions and comparing load 4 and load 1. Color bars indicate $\log _{10^{-}}$ transformed synchronization values in all plots. The box in the bottom right panel refers to the upper beta frequency range (19-25 $\mathrm{Hz}$ ) where significant linear load effects were observed.
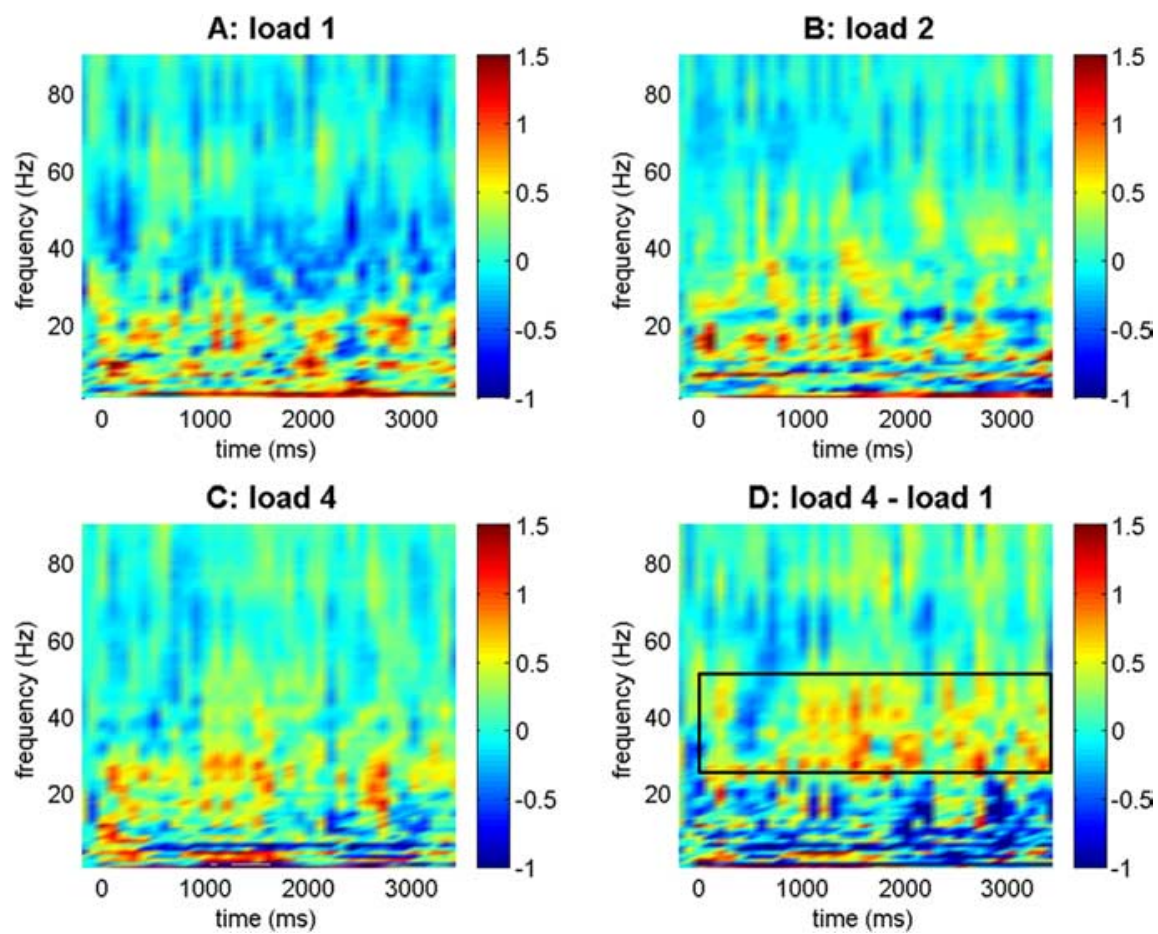

Figure 2. Synchronization between anterior parahippocampal gyrus and hippocampus. Baseline-normalized synchronization values are indicated for the different load conditions and comparing load 4 and load 1. Color bars indicate $\log _{10}$-transformed synchronization values in all plots. The box in the bottom right panel refers to the lower gamma frequency range $(26-50 \mathrm{~Hz})$ where significant linear load effects were observed.

Analyses in the separate bands revealed a significant load $\times$

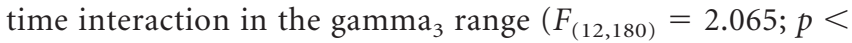
$0.05 ; \varepsilon=0.664)$, but no load effects and no load $\times$ time interaction in any other band. 

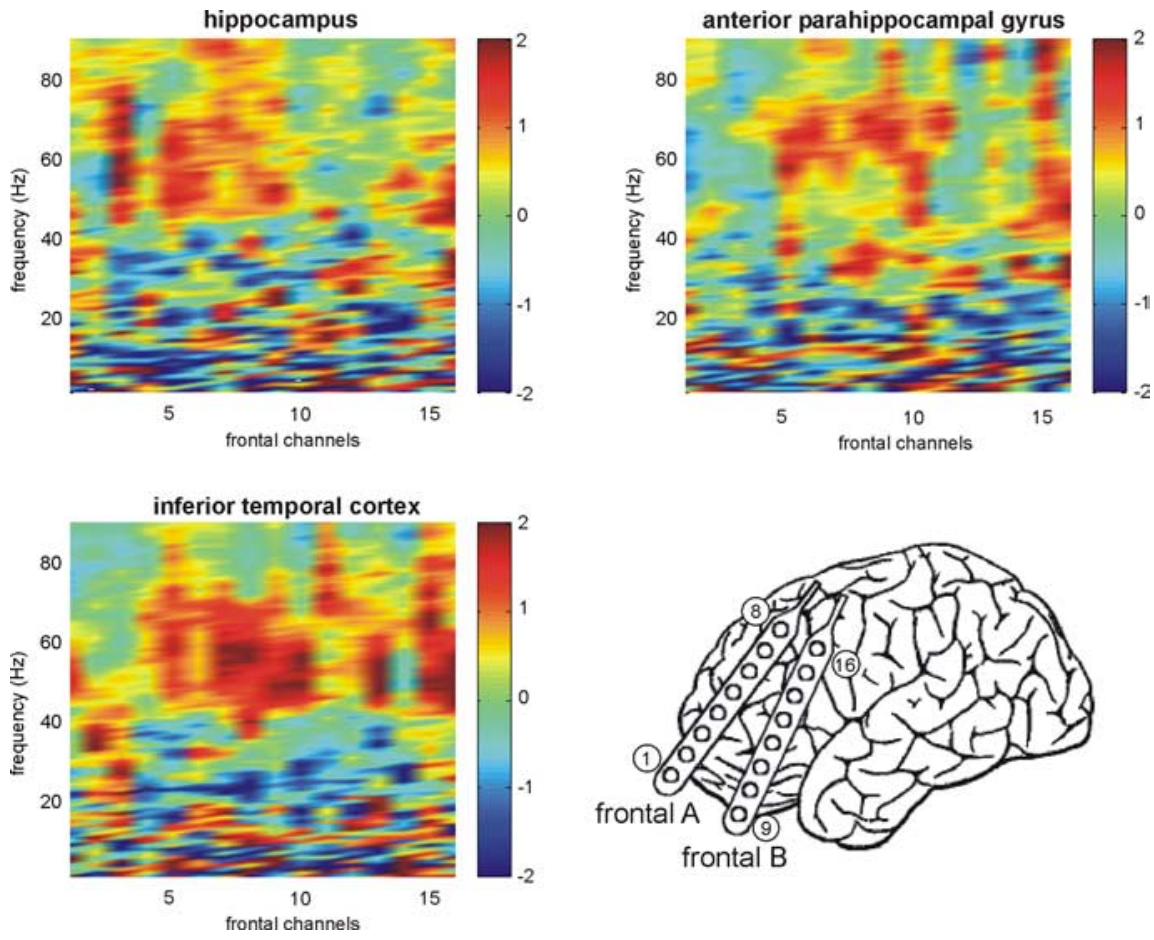

Figure 3. Synchronization with frontal contacts. Results from one patient with subdural frontal electrodes (diagram) and electrodes in the hippocampus, anterior parahippocampal gyrus, and inferior temporal cortex are shown: increased synchronization during maintenance of four items compared with one item. All regions showed enhanced synchronization in the upper gamma ranges, but this effect was most pronounced for frontal-inferior temporal synchronization.
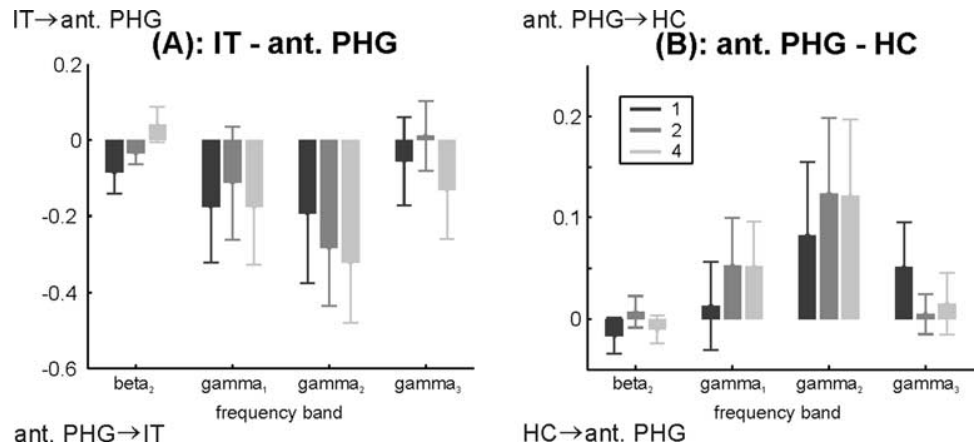

Figure 4. Directional coupling analysis. $A$, Effects of memory load on the direction of coupling between the anterior (ant.) parahippocampal gyrus and the IT cortex. We observed a significant load $\times$ band interaction $\left(F_{(2,10)}=3.014 ; p<0.05\right)$ and a trend for a load effect in the gamma 2 frequency range $\left(F_{(2,10)}=3.260 ; p<0.05\right)$. $B$, Effects of memory load on the direction of coupling between anterior parahippocampal gyrus and hippocampus. Although there was a trend for a load $\times$ band interaction $\left(F_{(6,66)}=2.104 ; p<0.05\right.$ ), coupling did not show a load effect in the separate bands (all $p$ values $>0.1$ ). Error bars indicate SEM.

\section{Directionality analysis}

Although the analysis of phase synchronization presented above indicates that coupling between the IT cortex and the aPHG and between aPHG and hippocampus increases with load in specific frequency bands, it does not allow to measure the direction of coupling. In other words, these results show enhanced communication between the IT cortex and the aPHG during multi-item $\mathrm{WM}$, but do not distinguish between an increased recruitment of the aPHG by the IT cortex (bottom-up influence) and an increased top-down modulation of processes within the IT cortex by the aPHG. To elucidate this question, we calculated a directionality index based on a phase-modeling approach (Rosenblum and Pikovsky, 2001) (see Materials and Methods). We determined the direction of coupling for higher-frequency bands starting at the beta ${ }_{2}$ range in which IT-aPHG synchronization increased significantly with load (Fig. $4 A$ ) (as described in Materials and Methods, the accuracy of the directionality index decreases for low frequencies). A two-way ANOVA with load and band as repeated measures revealed a significant load $X$ band interaction $\left(F_{(2,10)}=3.014 ; p<\right.$ $0.05)$, which was based on the increasing average values in the beta ${ }_{2}$ range and the decreasing average values in the gamma range. No main effects of load or band were observed. Subsequent separate oneway ANOVAs in the different frequency bands revealed a trend for a load effect in

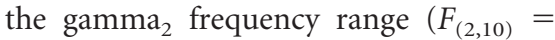
3.260; $p=0.081)$. In this band, average values decreased monotonically with load, consistent with the idea that multi-item WM requires increased top-down control of IT cortex activity by the aPHG. Between aPHG and hippocampus (Fig. 4B), we observed a trend for a load $\times$ band interaction $\left(F_{(6,66)}=2.104 ; p=0.071 ; \varepsilon=0.921\right)$, but no significant load effect in any separate band (all $p$ values $>0.1$ ). Finally, we tested whether the direction of coupling was significantly different from zero for each load condition, but these results did not reach significance (each $p>0.1$ ). This suggests bidirectional symmetric coupling between the respective structures (see Discussion).

\section{Functional connectivity of fMRI data}

In our functional connectivity analysis of fMRI data, we investigated regions showing differential connectivity with seed regions in the left MTL and the left PFC (see Materials and Methods) (Axmacher et al., 2007). Figure $5 A$ depicts the brain regions that were significantly correlated with the seed regions in the different load conditions compared with zero (one, two, or four items). Activity in the hippocampal seed was significantly correlated with activity in bilateral temporal regions, among them the ipsilateral IT cortex (fusiform gyrus) and the contralateral MTL. Activity in the prefrontal seed was significantly correlated with ipsilateral and contralateral frontal, temporal, and parietal regions, but there was no correlation with medial temporal activity. Supplemental Table 1 (available at www.jneurosci.org as supplemental material) provides an overview of all regions in which activity was significantly correlated with each of the two seed regions.

First, we investigated which specific regions showed loaddependent increases or decreases of functional connectivity with the seed regions (Table 1). With increasing load, there was a trend for an enhanced correlation of the hippocampal seed with a region in the visual association cortex (ipsilateral lingual gyrus; MNI coordinates, $-3,-65,3)$ and a decreased correlation with two contralateral frontal regions (middle and inferior frontal gyrus) and the contralateral superior temporal gyrus (Fig. 6A). 
There was no region showing increasing correlation with the prefrontal seed; however, there was a trend for a decreased correlation between the PFC and the cingulate gyrus with load (Fig. 6B).

Next, we tested the hypothesis that maintenance of an increasing number of items induces correlations of medial temporal and prefrontal activity with smaller and more selective networks. As described in Materials and Methods, we calculated the number of voxels showing significant correlation with the prefrontal and hippocampal seed regions in each subject and compared the different load conditions. An exemplary single-trial beta scatter plot obtained by correlating the MTL seed with a voxel in the left fusiform cortex (MNI coordinates, $-33,-55,-25)$ is shown in Figure $7 A$. The extension of both networks decreased with memory load (Fig. 7B): a two-way ANOVA with seed and load as repeated measures revealed a main effect of memory load $\left(F_{(2,44)}=5.69 ; p<0.01 ; \varepsilon\right.$ $=1.034)$, but no effect of seed $\left(F_{(1,22)}=\right.$ $1.95 ; p>0.1)$ and no seed $\times$ load interaction $\left(F_{(2,44)}=1.91 ; p>0.1 ; \varepsilon=1.065\right)$, indicating that load effects were similar for the regions correlated with the PFC and the MTL.

It might be argued that the extension of networks showing correlated activity with a voxel in the fusiform cortex should decrease as well, because sparser stimulus representations in this region would induce connectivity with more specific neocortical regions as well. However, the direct contrast of connectivity during processing of high and low load (Fig. 6A) revealed that functional connectivity between the hippocampal seed and the visual association cortex actually increased with load. Furthermore, the functional role of subregions within the IT cortex is probably more variable between subjects than the role of the MTL, so that load effects based on an IT seed might be difficult to find. To test these competing hypotheses, we also calculated the extension of networks showing significant correlation with a seed in the left fusiform cortex. The number of voxels showing correlation with this seed did not depend on load $\left(F_{(2,44)}\right.$ $=1.697 ; p=0.199)$. This also excludes the possibility that the effects of load observed for the prefrontal and medial temporal seed were unspecific.

Do the different connectivity measures in intracranial EEG and fMRI correlate with performance in the WM task? To investigate this question, we correlated behavioral performance measured as the number of correct responses (either averaged across load conditions or selectively in trials with four items) with (1) IT-aPHG synchronization, (2) aPHG-hippocampal synchronization, and (3) the number of voxels significantly correlated with the hippocampal seed. However, none of these correlations reached significance (each $p$ value $>0.2$ ). Another way to test for behavioral relevance of our measures would be to compare cor- rect and incorrect trials. However, from the 13 patients with intracranial EEG electrodes, only four had $>10$ incorrect trials in each load condition. This number is most likely insufficient for phase synchronization or directionality analyses. Similarly, for the fMRI subjects (who performed even better), only six had more than two trials for each condition. Thus, unfortunately, we could not test this interesting question, which should be addressed by future studies.

\section{Discussion}

\section{Phase-synchronization analysis}

The results of our phase-synchronization analysis indicate enhanced communication of content-specific regions in the IT cortex with the aPHG when multiple items are maintained simultaneously. We observed load-dependent synchronization increases between the IT cortex and aPHG in the upper beta range (19-25 $\mathrm{Hz}$ ) and between aPHG and hippocampus in the lower gamma range $(26-50 \mathrm{~Hz})$. A variety of studies suggest that the MTL is 
A MTL seed: load $4>$ load 1
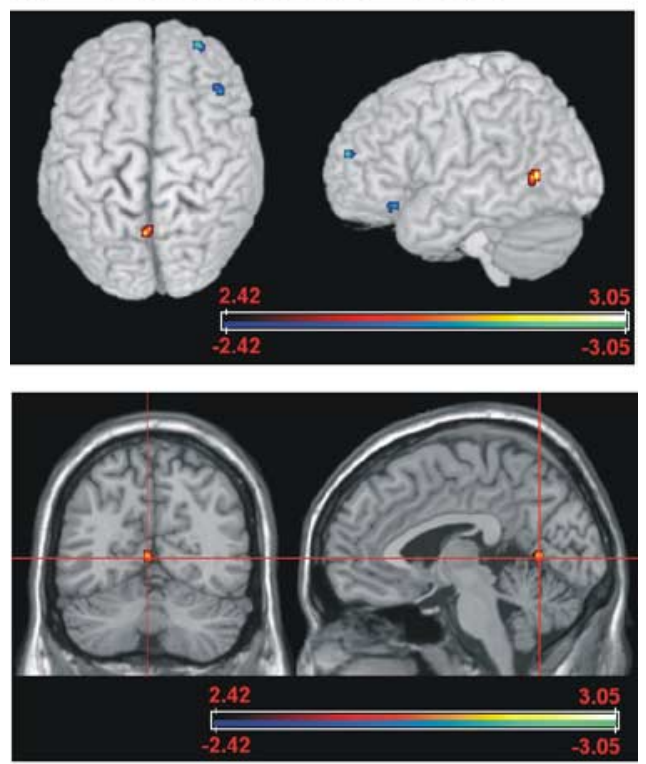

B $\quad$ PFC seed: load $4>\operatorname{load} 1$

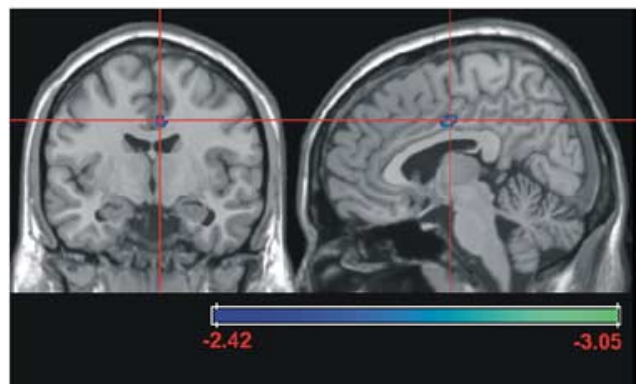

Figure 6. Load-dependent changes in functional connectivity. $\boldsymbol{A}$, Connectivity between the MTL seed and the ipsilateral lingual gyrus increased with memory load, whereas connectivity with regions in the contralateral inferior and middle frontal gyrus decreased. $\boldsymbol{B}$, Connectivity between the prefrontal seed and the cingulate gyrus decreased with memory load.

particularly well suited to support multi-item processing. Consecutive individual gamma cycles and cross-frequency coupling between gamma activity and low frequency oscillations have been suggested as mechanisms underlying representation of multiple items in WM (Palva et al., 2005; Jensen and Colgin, 2007), possibly constituting a so-called "multiplex buffer" (Lisman and Idiart, 1995; Jensen and Lisman, 2005). The enhanced gamma and beta synchronization between neural assemblies in the aPHG and the hippocampus, and between the IT and aPHG cortices during maintenance of an increasing number of items might correspond to such a mechanism, although additional analyses are necessary to directly test this idea.

To our knowledge, there are no previous data on synchronization with the MTL during WM. Using scalp EEG, effects of WM maintenance on neocortical synchronization were mainly found in the theta and alpha frequency ranges (Sarnthein et al., 1998; Sauseng et al., 2005a,b; Schack and Weiss, 2005; Mizuhara and Yamaguchi, 2007), consistent with the proposed role of thetaband activity for WM (Klimesch et al., 2005). This contrasts with the selective effects in higher-frequency ranges found in our data. We suggest two possible reasons for this apparent discrepancy. First, the spatial resolution of scalp EEG data is relatively low, tapping activity from larger neural assemblies. Larger assemblies are more often synchronized in lower frequency bands because of long axonal delays (von Stein and Sarnthein, 2000; Buzsaki, 2006). In contrast, the superior resolution of intracranial EEG recordings likely allows to record from smaller areas. Indeed, a previous intracranial EEG study of synchronization in the extrastriate cortex during WM maintenance found effects in the beta band (15-25 Hz) (Tallon-Baudry et al., 2001), similar to our data. In monkeys, beta synchronization in the IT cortex even depended on task performance (Tallon-Baudry et al., 2004). Second, previous studies suggest that gamma-band synchronization within the MTL plays an important role in LTM encoding (Fell et al., 2001), possibly via an effect on spike-timing dependent plasticity (Axmacher et al., 2006). Gamma-band activity has generally been related to the matching of stimulus information and stored memory patterns (Herrmann et al., 2004), and WM maintenance correlates with persistent increases of gamma power in intracranial EEG recordings (Howard et al., 2003; Axmacher et al., 2007).

\section{Directional coupling}

The direction of IT-aPHG and aPHG-hippocampal interactions was investigated by using a phase-modeling approach. During maintenance of an increasing number of items, the direction of coupling between the IT cortex and aPHG became more negative in the gamma ${ }_{2}$ frequency range, suggesting an increased topdown control of IT cortex activity by the aPHG. However, it should be noted that the directionality values in each load condition did not significantly differ from zero, indicating bidirectional (symmetric) coupling. Only a few previous studies investigated directional coupling of EEG data during cognitive tasks. Recently, Supp et al. (2007) used partial-directed coherence on source-reconstructed EEG data during object recognition and found widespread reciprocal information transfer with presentation of familiar objects, whereas unfamiliar objects elicited sparse and unidirectional coupling. Interestingly, they observed that coupling within the temporal lobe was driven by the IT cortex after presentation of individual unfamiliar items, reminiscent of our finding that the IT cortex drives the aPHG during maintenance of a single novel face.

What may be the function of the increased top-down direction of coupling from the aPHG to the IT cortex during multiitem WM? As described above, the MTL is in a unique position to support memory processes involving multiple items. During learning, it may convey an inhibitory signal to neocortical regions to render stimulus representations more sparse (Reddy and Kanwisher, 2006; Viskontas et al., 2006). This is consistent with our findings based on functional connectivity analysis of fMRI data, where we observed a decreased number of correlated voxels during multi-item WM, although we did not directly investigate regions responsible for representation of specific item types. However, it has to be noted that the different connectivity measures are not directly comparable (supplemental Discussion, available at www.jneurosci.org as supplemental material). Joint contributions of IT cortex and MTL have also been observed in an fMRI study during a delayed paired associate task: whereas initial stimulus maintenance depended on IT cortex activity, the MTL was additionally recruited during associative LTM retrieval (Ranganath et al., 2004). These findings support the idea that multi-item memory processing depends on the organization of item representations by the MTL. However, we did not find a significant effect of load on the direction of aPHG-hippocampal coupling, in line with the zero phase delay observed between these structures during LTM encoding (Fell et al., 2001). This symmetric coupling might suggest bidirectional information flow, consis- 
tent with our result that directionality of coupling did not differ significantly from zero; alternatively, it may indicate that aPHG-hippocampal coupling is driven by a third structure.

\section{fMRI data}

fMRI studies implementing analyses of functional connectivity usually investigate modifications in the correlation of blood oxygen level-dependent (BOLD) activity in a seed region with the activity in specific other regions. In our study, we also addressed this point and observed a loaddependent increase of functional connectivity between activity within the hippocampal seed region and a visual association area in the lingual gyrus. This result is consistent with previous findings by Rissman et al. (2008) who observed that connectivity between a hippocampal seed and the fusiform face area was found to increase with load. Increased corticohippocampal connectivity was also observed during successful LTM formation (Ranganath et al., 2005). These results further corroborate our iEEG findings that the hippocampus plays an increasing role in controlling activity in visual association cortex during maintenance of multiple items.

In addition to this more "conventional" application of the method by Rissman et al. (2004), we used functional connectivity of fMRI to study the extension of regions showing correlations with the seed regions in the different conditions. Although the calculation of the number of correlated voxels (instead of the analysis of connectivity with specific regions) might be an unusual approach to analyze the data, we do believe that it is useful to address a relevant question in our study, namely the size of regions showing correlated activity with the respective seed regions. This question requires to investigate extensive brain regions and can thus not be addressed with iEEG. One possible explanation for the decrease of the size of WM networks with increasing load has been already mentioned: regarding the functional connectivity with the hippocampal seed, it is possible that stimulus representations become more specific, and irrelevant activity is being suppressed. Although this interpretation is in accordance with the idea that item representations become more sparse during multi-item WM, similar to effects during LTM encoding (Reddy and Kanwisher, 2006; Axmacher et al., 2008) (supplemental Discussion, available at www.jneurosci.org as supplemental material), it is currently still speculative because we did not directly investigate item representations.

We observed similar effects in the networks based on seeds in the MTL and the PFC, which have both been proposed to support top-down modulation of activity in category specific visual regions during WM (Ranganath and D'Esposito, 2005). More specifically, some evidence suggests that they serve complementary roles for WM processes (Stern et al., 2001; Hasselmo and Stern, 2006): whereas the PFC appears to be predominantly important for WM maintenance of familiar items such as words, the MTL may play a role for maintenance of unfamiliar novel items. This division of labor might explain the apparent discrepancy between our findings and the results of Fiebach et al. (2006), who observed significant correlations between PFC and IT cortex during a WM paradigm with verbal (and therefore familiar) stimuli. However, the findings by Fiebach et al. (2006) are consistent with our results from phase synchronization in one patient with both pre-
B network extension

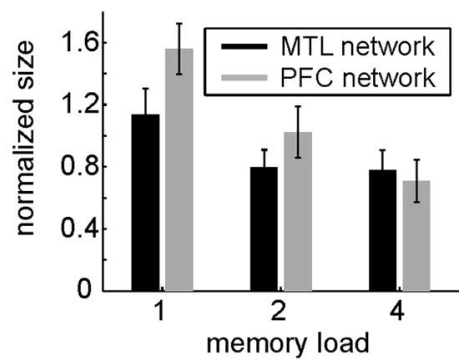

frontal and inferior temporal electrodes (Fig. 3). Possible differences between these methodologies are elaborated in the supplemental Discussion (available at www.jneurosci.org as supplemental material).

In previous univariate analyses of our fMRI data (Axmacher et al., 2007), we observed load effects in the MTL only in a region that is centered in the left hippocampus, but not in the aPHG, adjacent to the hippocampus. However, the spatial resolution of these functional imaging data (which are smoothed with a Gaussian filter of $8 \mathrm{~mm}$ half-maximum width) does not allow to unequivocally identify subregions within the MTL. It is thus possible that some of the activation in the cluster termed "hippocampus" is from anterior parahippocampal regions.

Together, WM maintenance of multiple items was associated with increased IT-aPHG and aPHG-hippocampal phase synchronization, with a reversal of the coupling direction from bottom-up to top-down, and with a decreased spatial extension of neural networks exhibiting correlated BOLD activity with the MTL and the PFC.

\section{References}

Aggleton JP, Shaw C, Gaffan EA (1992) The performance of postencephalitic amnesic subjects on two behavioural tests of memory: concurrent discrimination learning and delayed matching-to-sample. Cortex 28:359-372.

Allison T, Ginter H, McCarthy G, Nobre AC, Puce A, Luby M, Spencer DD (1994) Face recognition in human extrastriate cortex. J Neurophysiol 71:821-825.

Axmacher N, Mormann F, Fernández G, Elger CE, Fell J (2006) Memory formation by neuronal synchronization. Brain Res Rev 52:170-182.

Axmacher N, Mormann F, Fernández G, Cohen MX, Elger CE, Fell J (2007) Sustained neural activity patterns during working memory in the human medial temporal lobe. J Neurosci 27:7807-7816.

Axmacher N, Elger CE, Fell J (2008) Memory formation by refinement of neural representations: the inhibition hypothesis. Behav Brain Res 189:1-8.

Baddeley A, Hitch G (1974) Working memory. In: The psychology of learning and motivation, Vol VIII (Bower GH, ed), pp 47-89. New York: Academic.

Buzsaki G (2006) Rhythms of the brain. Oxford: Oxford UP.

Cohen NJ, Eichenbaum H (1993) Memory, amnesia, and the hippocampal system. Cambridge, MA: MIT.

Daubechies I (1990) The wavelet transform, time-frequency localization and signal analysis. IEEE Trans Inform Theory 36:961-1005.

Fell J, Klaver P, Lehnertz K, Grunwald T, Schaller C, Elger CE, Fernández G (2001) Human memory formation is accompanied by rhinalhippocampal coupling and decoupling. Nat Neurosci 4:1259-1264.

Fiebach CJ, Rissman J, D’Esposito M (2006) Modulation of inferotemporal cortex activation during verbal working memory maintenance. Neuron $51: 251-261$.

Fuster JM (1995) Memory in the cerebral cortex. Cambridge, MA: MIT. 
Hannula DE, Tranel D, Cohen NJ (2006) The long and the short of it: relational memory impairments in amnesia, even at short lags. J Neurosci 262:8352-8359.

Hasselmo ME, Stern CE (2006) Mechanisms underlying working memory for novel information. Trends Cogn Sci 10:487-493.

Herrmann CS, Munk MH, Engel AK (2004) Cognitive functions of gammaband activity: memory match and utilization. Trends Cogn Sci 8:347-355.

Hopf JM, Boehler CN, Luck SJ, Tsotsos JK, Heinze HJ, Schoenfeld MA (2006) Direct neurophysiological evidence for spatial suppression surrounding the focus of attention in vision. Proc Natl Acad Sci U S A 103:1053-1058.

Howard MW, Rizzuto DS, Caplan JB, Madsen JR, Lisman J, AschenbrennerScheibe R, Schulze-Bonhage A, Kahana MJ (2003) Gamma oscillations correlate with working memory load in humans. Cereb Cortex 13:1369-1374.

Huynh H, Feldt LS (1976) Estimation of the box correction for degrees of freedom from sample data in the randomized plot and split plot designs. J Educational Stat 1:69-82.

Jensen O, Colgin LL (2007) Cross-frequency coupling between neuronal oscillations. Trends Cogn Sci 11:267-269.

Jensen O, Lisman JE (2005) Hippocampal sequence-encoding driven by a cortical multi-item working memory buffer. Trends Neurosci 28:67-72.

Klimesch W, Schack B, Sauseng P (2005) The functional significance of theta and upper alpha oscillations. Exp Psychol 52:99-108.

Kralemann B, Cimponeriu L, Rosenblum M, Pikovsky A, Mrowka R (2007) Uncovering interaction of coupled oscillators from data. Phys Rev E Stat Nonlin Soft Matter Phys 76:0055201.

Lachaux JP, Rodriguez E, Martinerie J, Varela FJ (1999) Measuring phase synchrony in brain signals. Hum Brain Mapp 8:194-208.

Lisman JE, Idiart MA (1995) Storage of $7+/-2$ short-term memories in oscillatory subcycles. Science 267:1512-1515.

Mizuhara H, Yamaguchi Y (2007) Human cortical circuits for central executive function emerge by theta phase synchronization. Neuroimage 36:232-244.

Mormann F, Lehnertz K, David P, Elger CE (2000) Mean phase coherence as a measure for phase synchronization and its application to the EEG of epilepsy patients. Physica D 144:358-369.

Olson IR, Page K, Moore KS, Chatterjee A, Verfaellie M (2006) Working memory for conjunctions relies on the medial temporal lobe. J Neurosci 26:4596-4601

Osterhage H, Mormann F, Wagner T, Lehnertz K (2007) Measuring the directionality of coupling: phase versus state space dynamics and application to EEG time series. Int J Neur Sys 17:139-148.

Palva JM, Palva S, Kaila K (2005) Phase synchrony among neuronal oscillations in the human cortex. J Neurosci 25:3962-3972.

Petersson KM, Gisselgård J, Gretzer M, Ingvar M (2006) Interaction between a verbal working memory network and the medial temporal lobe. Neuroimage 33:1207-1217.

Piekema C, Kessels RP, Mars RB, Petersson KM, Fernández G (2006) The right hippocampus participates in short-term memory maintenance of object-location associations. Neuroimage 33:374-382.

Pikovsky AS, Rosenblum M, Kurths J (2001) Synchronization: a universal concept in nonlinear time sciences. Cambridge nonlinear time science series 12. Cambridge, UK: Cambridge UP.

Postle BR, Zarahn E, D’Esposito M (2000) Using event-related fMRI to assess delay-period activity during performance of spatial and nonspatial WM tasks. Brain Res Brain Res Protoc 5:57-66.

Ranganath C, D'Esposito M (2005) Directing the mind's eye: prefrontal, inferior and medial temporal mechanisms for visual working memory. Curr Opin Neurobiol 15:175-182.

Ranganath C, Cohen MX, Dam C, D’Esposito M (2004) Inferior temporal, prefrontal, and hippocampal contributions to visual working memory maintenance and associative memory retrieval. J Neurosci 24:3917-3925

Ranganath C, Heller A, Cohen MX, Brozinsky CJ, Rissman J (2005) Functional connectivity with the hippocampus during successful memory formation. Hippocampus 15:997-1005.

Reddy L, Kanwisher N (2006) Coding of visual objects in the ventral stream. Curr Opin Neurobiol 16:408-414.

Rissman J, Gazzaley A, D’Esposito M (2004) Measuring functional connectivity during distinct stages of a cognitive task. Neuroimage 23:752-763.

Rissman J, Gazzaley A, D’Esposito M (2008) Dynamic adjustments in prefrontal, hippocampal, and inferior temporal interactions with increasing visual working memory load. Cereb Cortex 18:1618-1629.

Rosenblum MG, Pikovsky AS (2001) Detecting direction of coupling in interacting oscillators. Phys Rev E Stat Nonlin Soft Matter Phys 64:045202.

Rosenblum MG, Cimponeriu L, Bezerianos A, Patzak A, Mrowka R (2001) Identification of coupling direction: application to cardiorespiratory interaction. Phys Rev E Stat Nonlin Soft Matter Phys 65:041909.

Sarnthein J, Petsche H, Rappelsberger P, Shaw GL, von Stein A (1998) Synchronization between prefrontal and posterior association cortex during human working memory. Proc Natl Acad Sci U S A 95:7092-7096.

Sauseng P, Klimesch W, Schabus M, Doppelmayr M (2005a) Frontoparietal EEG coherence in theta and upper alpha reflect central executive functions of working memory. Int J Psychophysiol 57:97-103.

Sauseng P, Klimesch W, Doppelmayr M, Pecherstorfer T, Freunberger R, Hanslmayr S (2005b) EEG alpha synchronization and functional coupling during top-down processing in a working memory task. Hum Brain Mapp 26:148-155.

Schack B, Weiss S (2005) Quantification of phase synchronization phenomena and their importance for verbal memory processes. Biol Cybern 92:275-287.

Smirnov D, Schelter B, Winterhalder M, Timmer J (2007) Revealing direction of coupling between neuronal oscillators from time series: phase dynamics modeling versus partial directed coherence. Chaos 17:013111.

Stern CE, Sherman SJ, Kirchhoff BA, Hasselmo ME (2001) Medial temporal and prefrontal contributions to working memory tasks with novel and familiar stimuli. Hippocampus 11:337-346.

Supp GG, Schlögl A, Trujillo-Barreto N, Müller MM, Gruber T (2007) Directed cortical information flow during human object recognition: analyzing induced EEG gamma-band responses in brain's source space. PLoS ONE 2:e684.

Tallon-Baudry C, Bertrand O, Fischer C (2001) Oscillatory synchrony between human extrastriate areas during visual short-term memory maintenance. J Neurosci 21:RC177.

Tallon-Baudry C, Mandon S, Freiwald WA, Kreiter AK (2004) Oscillatory synchrony in the monkey temporal lobe correlates with performance in a visual short-term memory task. Cereb Cortex 14:713-720.

Van Roost D, Solymosi L, Schramm J, van Oosterwyck B, Elger CE (1998) Depth electrode implantation in the length axis of the hippocamus for the presurgical evaluation of medial temporal lobe epilepsy: a computed tomography-based stereotactic insertion technique and its accuracy. Neurosurgery 43:819-826.

Viskontas IV, Knowlton BJ, Steinmetz PN, Fried I (2006) Differences in mnemonic processing by neurons in the human hippocampus and parahippocampal regions. J Cogn Neurosci 18:1654-1662.

von Stein A, Sarnthein J (2000) Different frequencies for different scales of cortical integration: from local gamma to long range alpha/theta synchronization. Int J Psychophysiol 38:301-313.

Witter MP, Amaral DG (1991) Entorhinal cortex of the monkey: V. Projections to the dentate gyrus, hippocampus, and subicular complex. J Comp Neurol 307:437-459.

Zarahn E, Aguirre G, D’Esposito M (1997) A trial-based experimental design for fMRI. Neuroimage 6:122-138. 